\title{
Accurate analytical approximation of asteroid deflection with constant tangential thrust
}

\author{
Claudio Bombardelli • Giulio Baù
}

\begin{abstract}
We present analytical formulas to estimate the variation of achieved deflection for an Earth-impacting asteroid following a continuous tangential low-thrust deflection strategy. Relatively simple analytical expressions are obtained with the aid of asymptotic theory and the use of Peláez orbital elements set, an approach that is particularly suitable to the asteroid deflection problem and is not limited to small eccentricities. The accuracy of the proposed formulas is evaluated numerically showing negligible error for both early and late deflection campaigns. The results will be of aid in planning future low-thrust asteroid deflection missions.
\end{abstract}

Keywords Asteroid deflection - Tangential thrust · Asymptotic expansion

\section{Introduction}

The subject of asteroid deflection is gaining increasing attention from the scientific community and is becoming a key topic in astrodynamics and space science. Starting in 1992, Ahrens and Harris first showed that Earth-impacting asteroids could be safely deflected by applying to the asteroid a small change in velocity $(\Delta V)$ sufficiently well before the expected impact. For a $\sim 100 \mathrm{~m}$ diameter asteroid the required $\Delta V$ could be obtained with a kinetic impactor of reasonable mass striking the asteroid with a relative velocity of a few $\mathrm{km} / \mathrm{s}$ a decade or more before the impact event. The preliminary results of Ahrens and Harris, obtained with a

C. Bombardelli (凶)

Space Dynamics Group, School of Aeronautics, Technical University of Madrid,

Plaza Cardenal Cisneros 3, Madrid, 28040, Spain

e-mail: claudio.bombardelli@upm.es

G. Baù

Department of Industrial Engineering, CISAS, University of Padova, Via Venezia 13, Padua, Italy 
simplified orbital model, were later refined by Carusi et al. in a series of three papers dealing with asteroid deflection by kinetic impact (Carusi 2005; Carusi et al. 2002; Carusi et al. 2008). Employing numerical and analytical techniques the previous authors computed the impulsive $\Delta V$ needed for deflecting an Earth-impacting asteroid on a generic orbit as a function of the epoch of interception and including the effect of gravitational scattering due to close approaches with the Earth and planets. In addition, they considered the option of multiple kinetic impactors (the "distributed deflection" approach) and analysed the requirements of a deflection mission taking into account the accessibility of the spacecraft terminal orbit from Earth given current launching constraints.

The kinetic impactor strategy, although often considered the easiest to implement, is only one of the many deflection methods proposed in the literature. The use of solar ablation (Melosh 1993; Vasile and Maddock 2010), gravitational pull (Lu and Love 2005; Gong et al. 2009) and ion beam irradiation Bombardelli and Peláez (2011) have been suggested as possible "contactless" low-thrust deflection methods which can gently modify the asteroid trajectory while eliminating the risk of fragmentation. Many subkilometre asteroids are thought to be rubble piles and could get easily disrupted by an impacting spacecraft (Carusi et al. 2008).

The computation of the asteroid deflection magnitude following a continuous low thrust acceleration can be demanding from a numerical point of view and approximate analytical formulas are highly desirable. For instance, a basic question that needs to be answered when dealing with low-thrust strategies is the influence of the different asteroid orbital parameters on the final achievable deflection. Similarly to the case of impulsive strategies, a first estimation of the achievable low-thrust deflection was developed for the case of an asteroid in circular orbit (Scheeres and Schweickart 2004) and assuming a constant continuous thrust phase followed by a coasting phase until the encounter epoch. A more detailed analysis was later provided by Izzo (Izzo 2007), who derived a semi-analytical quadrature formula, which can be used to compute a low-thrust deflection provided the time between the beginning of the manoeuvre and the expected impact epoch is sufficiently large. The formula was then employed to speed up a trajectory optimisation algorithm aimed at achieving maximum deflection given the available spacecraft mass that can be launched into orbit.

In a recent article (Bombardelli et al. 2011) a first-order analytical solution for the orbit evolution under constant tangential thrust was obtained with the aid of perturbation theory and a new set of orbital elements (Pelaez et al. 2007). The capability of representing both secular and periodic variations of the orbit position with any value of the orbit eccentricity and the high accuracy obtainable starting from weakly perturbed low-thrust orbits renders the method particularly suitable to the low-thrust asteroid deflection problem, in which applying a continuous tangential thrust is effective for changing the asteroid energy and maximise the deflection.

In the present article we exploit the above works in order to obtain a fully analytical low-thrust deflection formula capable of accurately computing the shift in the asteroid b-plane coordinates as a result of a continuous tangential thrust manoeuvre followed by a coasting phase. In order to keep the model and the equations as simple as possible we assume no close encounters with solar system planets occur between the beginning of the manoeuvre and the expected impact date and that the magnitude of the thrust (or drag) acceleration is constant.

The structure of the article is the following. First we compute analytical expressions to estimate the total b-plane asteroid deflection and minimum orbit intersection distance (MOID) as a function of the radial distance $(\Delta r)$ and time delay $(\Delta t)$ accumulated during the deflection and evaluated at the expected impact angular position of the asteroid orbit. In the subsequent section we provide analytical expressions to accurately compute $\Delta r$ and $\Delta t$ assuming a constant tangential thrust arc of given amplitude followed by a coasting are until the impact 
event. In the third section the accuracy of the complete deflection formulas obtained from the previous sections are evaluated by comparison with a full numerical solution. Finally simplified and compact formulas for both the total deflection and the MOID are obtained by accounting for dominant secular terms only.

\section{Kinematics of asteroid deflection}

In this section we will provide analytical expressions to quantify the achieved asteroid deflection given the accumulated variation of the position and velocity vector at the unperturbed impact event. In general terms we define impact event the outcome in which an asteroid and a selected point of interest in the Earth vicinity pass through a given region of space at the same instant of time. The selected point of interest can be, for example, just the Earth center, a point on the Earth surface, a resonant return keyhole (see Valsecchi et al. 2003) etc. While the present analysis is valid, with minor modifications, independently of what impact point is chosen, we will here refer to the case of an asteroid passing through the center of the Earth. In addition, we hereby make use of the term deffection to designate the minimum distance at which the asteroid will pass from the selected point of interest as a result of its trajectory being perturbed with some artificial means, hereby called deflection action, prior to the occurrence of the impact event. ${ }^{1}$

In the present section, the following simplifying assumptions will be made:

1. The asteroid orbital plane is invariant

2. The Earth orbit is assumed circular of radius $r_{E}=1 \mathrm{AU}$

3. The asteroid displacement from the impact point, as a result of the deflection action and any additional perturbation force, is small relatively to the radius of the Earth heliocentric orbit

4. No close encounters with the Earth or other planetary bodies occur from the beginning of the deflection action until prior to the impact event.

Let us employ as reference length and time units the Earth orbit radius $r_{E}$ and the reciprocal of the Earth mean motion $1 / \Omega_{E}$ and let $\langle X, Y, Z\rangle$ represent an inertial reference system with $X$ along the unperturbed asteroid orbit eccentricity vector, $Z$ orthogonal to the asteroid orbit plane and $Y$ following the right-hand rule.

The non-dimensional inertial position and velocity of the unperturbed asteroid with respect to $\langle X, Y, Z\rangle$ can be written as:

$$
\begin{aligned}
& \left(X_{A}, Y_{A}, Z_{A}\right)^{T}=\frac{p_{0}}{1+e_{0} \cos v}(\cos v, \sin v, 0)^{T}, \\
& \left(\dot{X}_{A}, \dot{Y}_{A}, \dot{Z}_{A}\right)^{T}=\frac{1}{\sqrt{p_{0}}}\left(-\sin v, e_{0}+\cos v, 0\right)^{T},
\end{aligned}
$$

where $e_{0}, v$ and $p_{0}$ are the eccentricity, true anomaly and dimensionless parameter of the initial asteroid orbit, respectively.

Let us suppose that for $v=\alpha$ the object is predicted to collide with the Earth. After denoting with $e_{0}$ and $p_{0}$ the asteroid eccentricity and dimensionless orbit parameter evaluated at the impact event the necessary condition for $\alpha$ in order to have an impact is:

$$
\alpha= \pm \cos ^{-1}\left(\frac{p_{0}-1}{e_{0}}\right)
$$

1 This should not be confused with the asteroid trajectory deflection, that is the distance between deflected and "undeflected" points on the asteroid trajectory measured at the same epoch. 
and a collision will be possible as long as the asteroid eccentricity $e_{0}$ and non-dimensional asteroid semimajor axis $a_{0}$ evaluated at the impact event satisfy:

$$
a_{0}\left(1-e_{0}\right)<1<a_{0}\left(1+e_{0}\right) \Leftrightarrow|\cos \alpha| \leq 1 .
$$

In Eq. (3) and in the remainder of the article the upper sign of the double sign notation refers to $0<\alpha<\pi$.

If we assume, with no loss of generality, that the predicted impact occurs at the asteroid ascending node with respect to the ecliptic, the Earth position with respect to $\langle X, Y, Z\rangle$ as a function of time $t$ measured from the the impact event can be written as:

$$
\begin{aligned}
\left(X_{E}, Y_{E}, Z_{E}\right)^{T}= & (\cos t \cos \alpha-\sin t \sin \alpha \cos i, \\
& \cos t \sin \alpha+\sin t \cos \alpha \cos i,-\sin t \sin i)^{T},
\end{aligned}
$$

where $i$ is the asteroid orbit inclination.

The corresponding Earth velocity results in:

$$
\begin{aligned}
\left(\dot{X}_{E}, \dot{Y}_{E}, \dot{Z}_{E}\right)^{T}= & (-\sin t \cos \alpha-\cos t \sin \alpha \cos i, \\
& -\sin t \sin \alpha+\cos t \cos \alpha \cos i,-\cos t \sin i)^{T} .
\end{aligned}
$$

Let $\boldsymbol{v}_{A}$ and $\boldsymbol{v}_{E}$ indicate, respectively, the unperturbed asteroid velocity vector and the Earth velocity vector both evaluated at the impact event $(v=\alpha, t=0)$. Using Eqs. $(2,3,5)$ $\boldsymbol{v}_{A}$ and $\boldsymbol{v}_{E}$ can be written in $\langle X, Y, Z\rangle$ axes as:

$$
\begin{aligned}
& \boldsymbol{v}_{A}=\left(\mp \frac{\sqrt{e_{0}^{2}-\left(p_{0}-1\right)^{2}}}{e_{0} \sqrt{p_{0}}}, \frac{e_{0}^{2}+p_{0}-1}{e_{0} \sqrt{p_{0}}}, 0\right)^{T}, \\
& \boldsymbol{v}_{E}=\left(\mp \frac{\sqrt{e_{0}^{2}-\left(p_{0}-1\right)^{2}}}{e_{0}} \cos i, \frac{p_{0}-1}{e_{0}} \cos i,-\sin i\right)^{T} .
\end{aligned}
$$

After excluding the case in which $\boldsymbol{v}_{A}$ and $\boldsymbol{v}_{E}$ are parallel, which will be dealt with in "Appendix I", let $\langle x, y, z\rangle$ represent an inertial reference system centered at the Earth-asteroid impact point and with axes directions defined as:

$$
\boldsymbol{u}_{x}=\frac{\boldsymbol{v}_{A}}{\left\|\boldsymbol{v}_{A}\right\|}, \quad \boldsymbol{u}_{z}=\frac{\boldsymbol{v}_{A} \wedge \boldsymbol{v}_{E}}{\left\|\boldsymbol{v}_{A} \wedge \boldsymbol{v}_{E}\right\|}, \quad \boldsymbol{u}_{y}=\boldsymbol{u}_{z} \wedge \boldsymbol{u}_{x}
$$

If we restrict ourselves to a small interval of time $\Delta t \ll 1$ around the impact event, we can consider the motion of both the Earth and the asteroid as uniform rectilinear with good approximation. In this hypothesis the trajectories of the two bodies are represented by two straight lines in the unperturbed $\langle x, y\rangle$ plane and intersecting with each other at the time of the impact.

If we now assume a deflection manoeuvre is applied to the asteroid by some artificial means or, in more general terms, the asteroid orbit is affected by an external perturbation, its velocity vector at the impact event can be considered virtually unchanged with respect to the unperturbed case. ${ }^{2}$ Conversely the asteroid position at the impact event will have shifted from the origin of $\{x, y, z\rangle$ to the point $(\Delta x, \Delta y, \Delta z)^{T}$. Under the uniform rectilinear motion assumption, the corresponding shift of the asteroid image in the b-plane can be determined with simple geometrical considerations as done in the following.

2 When close encounters with planetary bodies are excluded, as done here, typical velocity changes applied to asteroid are, at most, of the order of $\mathrm{cm} / \mathrm{s}$, completely negligible when compared to their heliocentric velocity. 


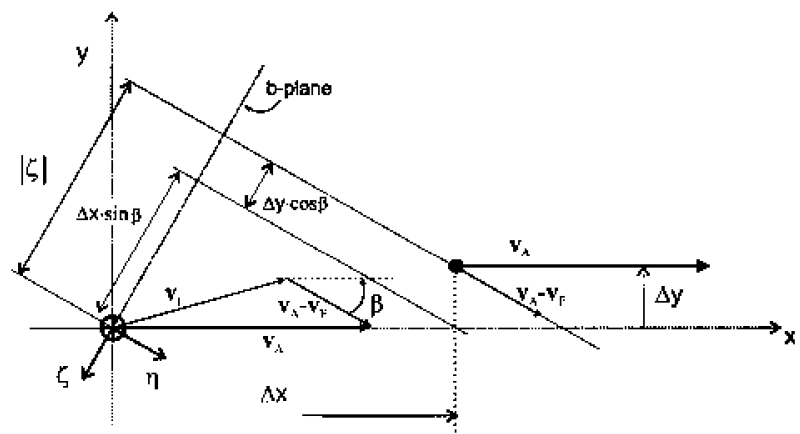

Fig. 1 Asteroid-Earth encounter geometry

First of all, following Valsecchi et al. (2003), we consider the $(\xi, \eta, \zeta)$ planetocentric b-plane frame in which the $\eta$ axis is directed along the asteroid velocity relative to the Earth, the $\zeta$ axis is in the direction opposite to the projection on the b-plane of the heliocentric velocity of the Earth and $\xi$ follows the right-hand rule. The $\xi$ axis, which as shown in the previous reference corresponds to the direction of the minimum orbit intersection distance (MOID), is orthogonal to the heliocentric Earth and asteroid velocity vectors and coincides with the previously defined $z$ axis.

With reference to Fig. 1 the image on the b-plane of a point $(\Delta x, \Delta y, \Delta z)^{T}$ obeys:

$$
\left\{\begin{array}{l}
\xi=-\Delta z \\
\zeta=-\Delta x \sin \beta-\Delta y \cos \beta
\end{array},\right.
$$

where $0<\beta \leq \pi$ is the angle between the heliocentric and geocentric velocity of the asteroid.

The total deflection results in:

$$
\delta=\sqrt{\xi^{2}+\zeta^{2}} .
$$

If the asteroid is deflected from a direct impact towards the Earth centre, the Earth gravitational effect can be added by noticing that $\delta$ is the distance between the Earth and the incoming trajectory asymptote. The real deflection is then the distance between the Earth and the vertex of the geocentric hyperbolic orbit and can be computed from the vis-viva integral (see for instance Battin 1999):

$$
d=\sqrt{\delta^{2}+a_{h}^{2}}-a_{h},
$$

where $a_{h}$ is the dimensionless semimajor axis of the asteroid hyperbolic trajectory:

$$
a_{h}=\frac{\mu_{E}}{\mu_{S} v_{\infty}^{2}}
$$

with $\mu_{E}$ and $v_{\infty}$ indicating the Earth gravitational parameter and the non-dimensional inbound relative velocity, respectively. The latter can be computed from Eqs. $(6,7)$ as:

$$
v_{\infty}=\left\|\boldsymbol{v}_{A}-\boldsymbol{v}_{E}\right\|=\sqrt{\frac{e_{0}^{2}-2 p_{0}^{3 / 2} \cos i+3 p_{0}-1}{p_{0}}} .
$$

Clearly, if $d$ is smaller than the Earth radius Eq. (10) is no longer meaningful.

One now needs to relate $\Delta x, \Delta y$ and $\Delta z$ to the characteristics of the perturbed orbital motion of the asteroid. Since the orbit plane is not affected by the deflection, when the asteroid 
reaches the impact angular position $\alpha$ it will have, in the most general case, an accumulated orbital radius variation $\Delta r$ and a time delay $\Delta t$ when compared to its original unperturbed trajectory. Because of the uniform rectilinear motion approximation, the accumulated time delay $\Delta t$ gives rise to a position shift along the velocity vector and its contribution can be written as:

$$
\Delta x_{1}=-v_{a s t} \Delta t
$$

where $v_{a s t}$ can be taken as the magnitude of the unperturbed heliocentric asteroid velocity at impact, which can be computed directly from Eq. (6) as:

$$
v_{\text {ast }}=\left\|\boldsymbol{v}_{A}\right\|=\sqrt{\frac{e_{0}^{2}-2 p_{0}-1}{p_{0}}} .
$$

On the other hand the variation $\Delta r$ affects in general all three components of the position shift as:

$$
\begin{gathered}
\Delta x_{2}=\Delta r\left(\boldsymbol{u}_{r} \cdot \boldsymbol{u}_{x}\right), \\
\Delta y=\Delta r\left(\boldsymbol{u}_{r} \cdot \boldsymbol{u}_{y}\right), \\
\Delta z=\Delta r\left(\boldsymbol{u}_{r} \cdot \boldsymbol{u}_{z}\right),
\end{gathered}
$$

where $\boldsymbol{u}_{r}=(\cos \alpha, \sin \alpha, 0)^{T}$ is the unit vector of the unperturbed asteroid position at the time of the impact and can be written as:

$$
\boldsymbol{u}_{r}=\left(\frac{p_{0}-1}{e_{0}}, \pm \sqrt{\frac{e_{0}^{2}-\left(p_{0}-1\right)^{2}}{e_{0}^{2}}}, 0\right)^{T}
$$

By employing Eqs. $(3,6,7)$ and after some algebraic simplifications Eq. (13) yields:

$$
\Delta x_{2}= \pm \Delta r \times \sqrt{\frac{e_{0}^{2}-\left(p_{0}-1\right)^{2}}{e_{0}^{2}+2 p_{0}-1}},
$$

so that the overall displacement along $x$ is:

$$
\Delta x=\Delta x_{1}+\Delta x_{2}=-\sqrt{\frac{e_{0}^{2}-2 p_{0}-1}{p_{0}}} \Delta t \pm \Delta r \sqrt{\frac{e_{0}^{2}-\left(p_{0}-1\right)^{2}}{e_{0}^{2}+2 p_{0}-1}} .
$$

Similarly, Eqs. $(14,15)$ can be put in the final form:

$$
\begin{aligned}
& \Delta y=\mp \Delta r \times \frac{p_{0} \sqrt{e_{0}^{2}-\left(p_{0}-1\right)^{2}} \cos i}{\sqrt{\left(e_{0}^{2}+2 p_{0}-1\right)\left(e_{0}^{2}+2 p_{0}-1-p_{0}^{2} \cos ^{2} i\right)}}, \\
& \Delta z=-\Delta r \times \frac{p_{0} \sin i}{\sqrt{e_{0}^{2}-p_{0}^{2} \cos ^{2} i+2 p_{0}-1}} .
\end{aligned}
$$

Note that for the case in which the asteroid orbit is circular $|\Delta z|=|\Delta r|, \Delta x=-v_{\text {ast }} \Delta t$ and $\Delta y=0$. 
Finally the angle $\beta$ can be expressed as:

$$
\begin{aligned}
& \cos \beta=\frac{\left(\boldsymbol{v}_{A}-\boldsymbol{v}_{E}\right) \cdot \boldsymbol{v}_{A}}{\left\|\boldsymbol{v}_{A}\right\|\left\|\boldsymbol{v}_{A}-\boldsymbol{v}_{E}\right\|}=\frac{e_{0}^{2}+2 p_{0}-1-p_{0}^{3 / 2} \cos i}{\sqrt{e_{0}^{2}+2 p_{0}-1} \sqrt{e_{0}^{2}+3 p_{0}-1-2 p_{0}^{3 / 2} \cos i}}, \\
& \sin \beta=\sqrt{1-\cos ^{2} \beta} .
\end{aligned}
$$

What is left to compute is now the accumulated encounter delay and radial variation, $\Delta t$ and $\Delta r$, for the perturbed asteroid orbit until the impact event following a given deflection strategy. It is important to remark that only the time delay contribution $\Delta x_{1}$ to the final deflection was considered in Izzo (2007). This would lead to a loss of accuracy in the deflection estimation, which would make the method not applicable to short warning time deflection as recognised by the same author.

\section{Optimal low thrust deflection strategy}

When reasonable warning time is available, that is, the deflection manoeuvre starts sufficiently early in time with respect to the actual predicted impact, the most effective way to deflect an asteroid is to modify the semimajor axis (or, equivalently, the energy) of the asteroid orbit (Ahrens and Harris 1992) hence obtaining a secular growth of the time delay at encounter and, in turns, a secular shift along the $\zeta$ axis of the Earth encounter b-plane. In other words, the phasing term $v_{a s t} \Delta t$, related to the orbit energy variation, is dominant over the asteroid orbit radial variation $\Delta r$ provided the deflection manoeuvre is initiated sufficiently early in time.

Ultimately, one would need to determine the thrust steering angle needed to obtain the maximum increase (or decrease) in orbit semimajor axis. For the low-thrust asteroid deflection case, in which the orbital elements vary by an extremely small amount, such optimum condition corresponds to having the thrust vector virtually tangent to the orbit at all times (Song et al. 2007).

Given these considerations, we will assume, for the present work, the asteroid undergoes a continuous tangential thrust phase followed by a coasting phase until the predicted impact event. During the thrust are the acceleration will be kept constant hence allowing to make use of a recently published analytical solution for the constant tangential thrust acceleration along a generic orbit (Bombardelli et al. 2011).

It is worth pointing out that this assumption is compatible with low thrust deflection missions based on nuclear electric propulsion. When solar-powered deflection methods are considered a thrust magnitude varying with the inverse square distance from the Sun would be more realistic (Colombo et al. 2009).

\section{Dynamics of asteroid deflection under constant tangential thrust}

In this section we will provide accurate analytical expressions to estimate the accumulated radial variation $\Delta r$ and time delay $\Delta t$ following a purely tangential deflection manoeuvre with constant thrust acceleration. The problem is formulated as follows.

A continuous tangential thrust acceleration of constant magnitude is applied to the asteroid across the trajectory arc $\left[\theta_{0}, \theta_{1}\right]$ with $\theta$ denoting the asteroid angular position measured from its initial eccentricity vector. In the general case the thrust can be applied over multiple 
revolutions. All orbital perturbations other than the deflection acceleration are neglected. Following the thrust phase the asteroid is left on its final osculating orbit, where all external perturbations are neglected, until it enters the sphere of influence of the Earth. The coasting trajectory arc from the end of the thrust manoeuvre until Earth approach is denoted with $\left[\theta_{1}, \theta_{2}=\alpha\right]$.

\subsection{Radial displacement}

Following Bombardelli et al. (2011) the asteroid trajectory can be characterised by the Peláez generalised orbital parameters $q_{1}, q_{2}, q_{3}$ defined as:

$$
\begin{aligned}
& q_{1}=\frac{e}{h} \cos \Delta \gamma, \\
& q_{2}=\frac{e}{h} \sin \Delta \gamma, \\
& q_{3}=\frac{1}{h},
\end{aligned}
$$

where $h$ is the dimensionless angular momentum of the osculating orbit, $e$ its eccentricity and $\Delta \gamma$ is, for the constant orbit plane case, the rotation of the eccentricity vector with respect to its initial value. Here, unlike Sect. 2, the reference units for length and time are, respectively, the asteroid pericentre radius $R_{p 0}$ at the beginning of the thrust manoeuvre and the reciprocal $1 / \Omega_{0}$ of the angular rate of a circular orbit with radius equal to $R_{p 0}$ :

$$
\Omega_{0}=\sqrt{\frac{\mu_{S}}{R_{p 0}^{3}}} .
$$

In order to avoid confusion we will indicate with $\rho$ and $\tau$, respectively, the orbit radius and time when using $R_{p 0}$ and $1 / \Omega_{0}$ as reference units.

The eccentricity vector magnitude and rotation can be related to the generalised orbital parameters, for later use, using Eqs. $(22,23)$ :

$$
\begin{array}{r}
e=\frac{\sqrt{q_{1}^{2}+q_{2}^{2}}}{q_{3}}, \\
\Delta \gamma=\tan ^{-1} \frac{q_{2}}{q_{1}} .
\end{array}
$$

The instantaneous orbit radius $\rho$ is a function of the angular position $\theta$ and the three generalised orbital parameters $q_{i}$ as follows (Bombardelli et al. 2011):

$$
\rho=\left(q_{3}^{2}+q_{1} q_{3} \cos \theta+q_{2} q_{3} \sin \theta\right)^{-1} .
$$

Here we are interested in the radius variation $\Delta \rho$ between the unperturbed and actual trajectory evaluated at the impact point angular position $\alpha$, that is:

$$
\Delta \rho=\left(q_{3}^{2}+q_{1} q_{3} \cos \alpha+q_{2} q_{3} \sin \alpha\right)^{-1}-\left(q_{30}^{2}+q_{10} q_{30} \cos \alpha+q_{20} q_{30} \sin \alpha\right)^{-1},
$$

where $q_{i 0}$ are the generalised orbital element of the unperturbed trajectory of eccentricity $e_{0}$ :

$$
\begin{aligned}
& q_{10}=\frac{e_{0}}{\sqrt{1+e_{0}}}, \\
& q_{20}=0, \\
& q_{30}=\frac{1}{\sqrt{1+e_{0}}} .
\end{aligned}
$$


The evolution of $q_{1}, q_{2}, q_{3}$ along the thrust arc [ $\left.\theta_{0}, \theta_{1}\right]$ obeys (Bombardelli et al. 2011):

$$
\begin{aligned}
& q_{1}=q_{10}+\epsilon q_{11}+o(\epsilon), \\
& q_{2}=q_{20}+\epsilon q_{21}+o(\epsilon), \\
& q_{3}=q_{30}+\epsilon q_{31}+o(\epsilon),
\end{aligned}
$$

where $\epsilon$ is the ratio between the tangential acceleration and the local gravitational acceleration at the beginning of the manoeuvre:

$$
\epsilon=\frac{F_{t} / m_{a s t}}{\mu_{S} / R_{p 0}^{2}},
$$

with $F_{t}$ and $m_{\text {ast }}$ indicating the thrust magnitude and asteroid mass, respectively.

After substituting Eq. (3) and Eqs. (31-33) into Eq. (27) and expanding in Taylor series for small $\epsilon$ we obtain:

$$
\begin{aligned}
\Delta \rho= & \frac{\epsilon\left(1+e_{0}\right)^{3 / 2}}{e_{0} p_{0}^{2}} \\
& \times\left[\left(1-p_{0}\right) q_{11} \mp q_{21} \sqrt{e_{0}^{2}-\left(p_{0}-1\right)^{2}}-e_{0}\left(p_{0}+1\right) q_{31}\right]+o(\epsilon) .
\end{aligned}
$$

The terms $q_{i 1}$ appearing in the above equation are given in Bombardelli et al. (2011) as:

$$
\begin{aligned}
& q_{11}=\frac{Q_{11}\left(E_{1}\right)-Q_{11}\left(E_{0}\right)}{\left(1+e_{0}\right)^{1 / 2}\left(1-e_{0}\right)^{2}}, \\
& q_{21}=\frac{Q_{21}\left(E_{1}\right)-Q_{21}\left(E_{0}\right)}{\left(1-e_{0}\right)^{3 / 2}}, \\
& q_{31}=\frac{Q_{31}\left(E_{1}\right)-Q_{31}\left(E_{0}\right)}{\left(1+e_{0}\right)^{1 / 2}\left(1-e_{0}\right)^{2}},
\end{aligned}
$$

where $E_{0}, E_{1}$ are the values, at the beginning and at the end of the thrust arc, of the unperturbed orbit eccentric anomaly, which is related to the orbit angular position $\theta$ through the equality:

$$
\tan \frac{E}{2}=\sqrt{\frac{1-e_{0}}{1+e_{0}}} \tan \frac{\theta}{2} .
$$

The functions $Q_{i 1}$ can be split into a secular and an oscillatory component:

$$
Q_{i 1}(E)=Q_{i 1, \sec }(E)+Q_{i 1, \text { osc }}(E) .
$$

The secular component yields:

$$
\begin{aligned}
& Q_{11, \sec }(E)=k_{1} E, \\
& Q_{21, \sec }(E)=0, \\
& Q_{31, \sec }(E)=k_{3} E,
\end{aligned}
$$

where $k_{1}$ and $k_{3}$ are given by:

$$
\begin{aligned}
& k_{1}=\frac{2 \mathcal{E}\left(e_{0}\right)\left(2-e_{0}^{2}\right)-4 \mathcal{K}\left(e_{0}\right)}{\pi e_{0}}, \\
& k_{3}=\frac{2 \mathcal{E}\left(e_{0}\right)-4 \mathcal{K}\left(e_{0}\right)}{\pi},
\end{aligned}
$$


with $\mathcal{K}$ and $\mathcal{E}$ indicating complete elliptic integrals of the first and second kind, respectively:

$$
\begin{aligned}
\mathcal{K}\left(e_{0}\right) & =\int_{0}^{1} \frac{d z}{\sqrt{\left(1-z^{2}\right)\left(1-e_{0}^{2} z^{2}\right)}}, \\
\mathcal{E}\left(e_{0}\right) & =\int_{0}^{1} \sqrt{\frac{1-e_{0}^{2} z^{2}}{1-z^{2}}} d z .
\end{aligned}
$$

The oscillatory terms, whose closed-form solution in terms of incomplete elliptic integrals is given in Bombardelli et al. (2011) can be written in the compact matrix form:

$$
\begin{aligned}
& Q_{11, \text { os } c}(E)=\left(\mathcal{Q}_{1} \boldsymbol{v}_{e 0}\right)^{T} \boldsymbol{v}_{S}, \\
& Q_{21, \text { osc }}(E)=\left(\mathcal{Q}_{2} \boldsymbol{v}_{e 0}\right)^{T} \boldsymbol{v}_{C}, \\
& Q_{31, \text { os } c}(E)=\left(\mathcal{Q}_{3} \boldsymbol{v}_{e 0}\right)^{T} \boldsymbol{v}_{S},
\end{aligned}
$$

where:

$$
\begin{aligned}
\boldsymbol{v}_{e 0} & =\left(1, e_{0}, e_{0}^{2}, e_{0}^{3} \ldots\right)^{T}, \\
\boldsymbol{v}_{S} & =(\sin E, \sin 2 E, \sin 3 E, \ldots)^{T}, \\
\boldsymbol{v}_{C} & =(\cos E, \cos 2 E, \cos 3 E, \ldots)^{T},
\end{aligned}
$$

and the $8 \times 8 \mathcal{Q}_{i}$ matrices are given in "Appendix II" (published as Electronic Supplementary Material).

\subsection{Time delay}

The total time delay at the impact angular position $\alpha$ is made up by one contribution $\Delta \tau_{01}$ accumulated during the thrust phase, and a second contribution $\Delta \tau_{12}$ accumulated throughout the coasting phase due to the variation of the orbit semimajor axis when compared to the unperturbed trajectory.

Following Bombardelli et al. (2011), the first contribution can be written as:

$$
\Delta \tau_{01}=\epsilon \frac{T\left(E_{1}\right)-T\left(E_{0}\right)}{\left(1-e_{0}\right)^{9 / 2}\left(1+e_{0}\right)} .
$$

In the above equation the function $T$ can be expressed as a sum of secular and oscillatory terms:

$$
T(E)=T_{s e c}(E)+T_{o s c}(E) .
$$

The secular part yields (Bombardelli et al. 2011):

$$
\begin{aligned}
T_{\sec }(E)= & \frac{3}{2}\left(k_{1} e_{0}-k_{3}\right) E^{2}+E\left[\left(k_{3} e_{0}\left(5-e_{0}^{2}\right)-2 k_{1}\left(1+e_{0}^{2}\right)\right) \sin E\right. \\
& \left.+\frac{1}{2} e_{0}\left(k_{1}-k_{3} e_{0}\right) \sin 2 E+g\left(e_{0}, E_{0}\right)\right]
\end{aligned}
$$

where:

$$
g\left(e_{0}, E_{0}\right)=\left(\boldsymbol{G} \boldsymbol{v}_{e 0}\right)^{T} \boldsymbol{w}_{S 0}
$$

with:

$$
\boldsymbol{w}_{S 0}=\left(1, \sin E_{0}, \sin 2 E_{0}, \sin 3 E_{0}, \ldots\right)^{T},
$$


and the $9 \times 9 \boldsymbol{G}$ matrix is given in "Appendix II" (published as Electronic Supplementary Material).

Finally, the oscillatory part yields (Bombardelli et al. 2011):

$$
\begin{aligned}
T_{O S C}(E)= & \left(\boldsymbol{H} \boldsymbol{v}_{e 0}\right)^{T} \boldsymbol{v}_{C}+\left(\boldsymbol{P}_{1} \boldsymbol{v}_{e 0}\right)^{T} \boldsymbol{v}_{C 0} \cos E \\
& +\left(\boldsymbol{P}_{2} \boldsymbol{v}_{e 0}\right)^{T} \boldsymbol{v}_{S 0} \sin E+\left(\boldsymbol{P}_{3} \boldsymbol{v}_{e 0}\right)^{T} \boldsymbol{v}_{C 0} \cos 2 E+\left(\boldsymbol{P}_{4} \boldsymbol{v}_{e 0}\right)^{T} \boldsymbol{v}_{S 0} \sin 2 E,(44)
\end{aligned}
$$

where:

$$
\boldsymbol{v}_{S 0}=\boldsymbol{v}_{S}\left(E=E_{0}\right) ; \boldsymbol{v}_{C 0}=\boldsymbol{v}_{C}\left(E=E_{0}\right),
$$

and the $\boldsymbol{H}$ and $\boldsymbol{P}_{i}$ matrices are given in "Appendix II" (published as Electronic Supplementary Material).

The time delay $\Delta \tau_{12}$ accumulated during the coasting phase is the difference in time traveled between the same arc $\Delta \theta_{c}$ of two Keplerian orbits having different semimajor axis, eccentricity and argument of periapsis:

$$
\Delta \tau_{12}=\frac{T_{k e p, 1}\left(\hat{E}_{2}\right)-T_{k e p, 1}\left(\hat{E}_{1}\right)}{q_{3}^{3}\left(1-e_{1}^{2}\right)^{3 / 2}}-\frac{T_{k e p, 0}\left(E_{2}\right)-T_{k e p, 0}\left(E_{1}\right)}{q_{30}^{3}\left(1-e_{0}^{2}\right)^{3 / 2}},
$$

where the functions $T_{k e p, i}$ are defined as:

$$
\begin{gathered}
T_{k e p, 0}(E)=E-e_{0} \sin E, \\
T_{k e p, 1}(E)=E-e_{1} \sin E,
\end{gathered}
$$

$e_{1}$ is the orbit eccentricity at the end of the thrust arc, and $\hat{E}$ is the final osculating orbit eccentric anomaly, whose relationship with the arc angle $\theta$ is:

$$
\tan \frac{\hat{E}}{2}=\sqrt{\frac{1-e_{1}}{1+e_{1}}} \tan \frac{\left(\theta-\Delta \gamma_{1}\right)}{2} .
$$

In the above equation $\Delta \gamma_{1}$ is the rotation of the eccentricity vector at the end of the thrust arc.

Equation (45) can be written in compact form by expressing $e_{1}$ and $\Delta \gamma_{1}$ as a function of the parameters $q_{i}$ [Eqs. $\left.(25,26)\right]$ and by taking into account the expansions (31-33). After expanding in Taylor series and performing algebraic simplifications we obtain:

$$
\begin{aligned}
\Delta \tau_{12}= & \frac{\epsilon}{\left(1-e_{0}\right)^{5 / 2}\left(1+e_{0}\right)^{1 / 2}} \\
& \times\left[3\left(e_{0} q_{11}-q_{31}\right)\left(E_{2}-E_{1}\right)+q_{11}\left(\mathbf{S}_{1} \boldsymbol{v}_{e 0}\right)^{T}\left(\boldsymbol{v}_{S 2}-\boldsymbol{v}_{S 1}\right)\right. \\
& \left.+q_{21}\left(\mathbf{S}_{2} \boldsymbol{v}_{e 0}\right)^{T}\left(\boldsymbol{v}_{C 2}-\boldsymbol{v}_{C 1}\right)+q_{31}\left(\mathbf{S}_{3} \boldsymbol{v}_{e 0}\right)^{T}\left(\boldsymbol{v}_{S 2}-\boldsymbol{v}_{S 1}\right)\right]+o(\epsilon),
\end{aligned}
$$

where:

$$
\boldsymbol{v}_{S i}=\boldsymbol{v}_{S}\left(E=E_{i}\right) ; \boldsymbol{v}_{C i}=\boldsymbol{v}_{C}\left(E=E_{i}\right),
$$

and the $\boldsymbol{S}_{i}$ matrices are given as Supplementary Material to this article.

Finally, the radial variation and accumulated time delay at the impact event, as to be used in Eqs. (17-19), ultimately yield:

$$
\begin{aligned}
& \Delta r=\frac{R_{p 0} \Delta \rho}{r_{E}}=\frac{p_{0}}{1+e_{0}} \Delta \rho, \\
& \Delta t=\frac{\Omega_{E}\left(\Delta \tau_{01}+\Delta \tau_{12}\right)}{\Omega_{0}}=\left(\frac{p_{0}}{1+e_{0}}\right)^{3 / 2}\left(\Delta \tau_{01}+\Delta \tau_{12}\right) .
\end{aligned}
$$




\section{Analytical deflection formula}

The evolution (measured in $\mathrm{AU}$ ) of the asteroid image in the encounter b-plane $(\xi, \zeta)$ and the total deflection magnitude $\delta$ can now be summarised, by use of Eqs. $(8,9,17-19,20,21)$, as follows:

$$
\begin{aligned}
& \left\{\begin{array}{l}
\xi=C_{\xi r} \Delta r \\
\zeta=C_{\zeta t} \Delta t+C_{\zeta r} \Delta r
\end{array}\right. \\
& \delta=\sqrt{\xi^{2}+\zeta^{2}}
\end{aligned}
$$

where:

$$
\begin{aligned}
C_{\xi r}= & \frac{p_{0} \sin i}{\sqrt{e_{0}^{2}-p_{0}^{2} \cos ^{2} i+2 p_{0}-1}}, \\
C_{\zeta t}= & \sqrt{\frac{e_{0}^{2}-p_{0}^{2} \cos ^{2} i+2 p_{0}-1}{e_{0}^{2}-2 p_{0}^{3 / 2} \cos i+3 p_{0}-1}} \\
C_{\zeta r}= & \pm \frac{\sqrt{p_{0}\left[e_{0}^{2}-\left(p_{0}-1\right)^{2}\right]}}{\left(e_{0}^{2}+2 p_{0}-1\right) \sqrt{e_{0}^{2}-2 p_{0}^{3 / 2} \cos i+3 p_{0}-1}} \\
& \times\left[\frac{\sqrt{p_{0}} \cos i\left(e_{0}^{2}-p_{0}^{3 / 2} \cos i+2 p_{0}-1\right)}{\sqrt{e_{0}^{2}-p_{0}^{2} \cos i+2 p_{0}-1}}-\sqrt{e_{0}^{2}-p_{0}^{2} \cos ^{2} i+2 p_{0}-1}\right] .
\end{aligned}
$$

and where the dimensional radial variation $\Delta r$ and time delay $\Delta t$ are given through Eqs. (35, 48 ) and (49) whose different terms can be found in the previous section. It is worth pointing out that the deflection component along the $\xi$ axis [Eqs. $(50,52)]$ is equivalent to the analytical MOID, or AMOID, computed by Bonanno (2000).

The advantage of the closed-form analytical formulas when compared with a full numerical integration can be appreciated from Bombardelli et al. (2011) where an order of magnitude of computation time difference was obtained. Furthermore, unlike the case discussed in Bombardelli et al. (2011) when dealing with asteroid deflection, characterised by an extremely small value of the dimensionless thrust acceleration, no rectification of the analytical propagation is required and the difference in computation efficiency is expected to further increase.

A more compact, approximated expression of the achieved deflection can be obtained by considering only the $\zeta$ axis component of the deflection and retaining only the dominant secular terms. In this way only the phasing terms $\Delta t$ contributes to the deflection whose final expression, measured in $\mathrm{AU}$, yields:

$$
\begin{aligned}
\delta \approx & \frac{3 F_{t} / m_{\text {ast }}}{2 \mu_{S} / r_{E}^{2}} \\
& \times \frac{p_{0}^{7 / 2}\left(e_{0}^{2}-p_{0}^{2} \cos ^{2} i+2 p_{0}-1\right)^{1 / 2}\left(k_{1} e_{0}-k_{3}\right)\left(E_{1}-E_{0}\right)\left(2 E_{2}-E_{1}-E_{0}\right)}{\left(1-e_{0}^{2}\right)^{9 / 2}\left(e_{0}^{2}-2 p_{0}^{3 / 2} \cos i+3 p_{0}-1\right)^{1 / 2}} .
\end{aligned}
$$


Table 1 Main characteristics of the two chosen asteroids

\begin{tabular}{lll}
\hline & $2007 \mathrm{VK}_{184}$ & $2011 \mathrm{AG}_{5}$ \\
\hline Mass (kg) & $3.3 \times 10^{9}$ & $3.9 \times 10^{9}$ \\
Diameter (m) & 130 & 140 \\
Semimajor axis (AU) & 1.726 & 1.43 \\
Eccentricity & 0.57 & 0.39 \\
Inclination $\left({ }^{\circ}\right)$ & 1.22 & 3.68 \\
Period (days) & 828 & 625 \\
\hline
\end{tabular}

Similarly, an approximate expression can be obtained for the MOID by neglecting oscillatory terms:

$$
\xi \approx \frac{F_{t} / m_{a s t}}{\mu_{S} / r_{E}^{2}} \times \frac{p_{0}^{2} \sin i\left[\left(1-p_{0}\right) k_{1}-e_{0}\left(1+p_{0}\right) k_{3}\right]\left(E_{1}-E_{0}\right)}{e_{0}\left(1-e_{0}^{2}\right)^{2}\left(e_{0}^{2}-p_{0}^{2} \cos ^{2} i+2 p_{0}-1\right)^{1 / 2}} .
$$

Note however that for the case of the MOID computation oscillatory terms are usually not negligible even for asteroids with low eccentricities so Eq. (56) should only be used for order-of-magnitude estimates.

The accuracy of the above formulas was tested by comparison with a very accurate numerical integrator and considering a deflection initiated up to 10 years before the impact event of the two asteroids $2007 \mathrm{VK}_{184}$ and $2011 \mathrm{AG}_{5}$, currently the only two known asteroids with index 1 in the Torino scale (Table 1). The former will pass at close distance with the Earth in 2048 with no close approaches with the Earth or other planets between 2014 and 2048. The latter will come close to our planet in 2040 with no close planetary approaches between 2028 and 2040 (as retrieved from the NEODyS website). For each asteroid two deflection strategies have been considered. For the first strategy a continuous tangential thrust of $1 \mathrm{~N}$ is applied continuously starting from 10 years before the scheduled impact. In the second strategy a $1 \mathrm{~N}$ thrust is applied continuously for 2 years with a subsequent coasting trajectory of up to 8 years.

Figure 2 represents the numerically-computed deflection magnitude $\delta$ and MOID $\xi$ showing, as already known in the literature, that the latter can be more than two orders of magnitudes smaller when the deflection begins sufficiently far back in time (say more than 2 years). This fact is also reflected by the representation of the trajectory of the asteroid impact point in the b-plane as the deflection starting point moves back in time from the predicted impact (Fig. 3). As it can be seen, following an initial oscillation, the $\zeta$ component starts to rapidly increase becoming dominant over the $\xi$ component already after half an asteroid orbital period.

Finally, the relative error of the full and approximate analytical deflection formula is plotted in Fig. 4. The full analytical formula exhibits a remarkable accuracy with less than $0.02 \%$ relative error in all cases when including oscillatory terms up to order 20 . The relative error is still very small (always less than $0.1 \%$ except for deflection manoeuvres starting shortly before the impact) using an order 8th expansion. The approximate formula of Eq. (55) works quite well (less than $10 \%$ relative error) for deflection campaigns longer than one asteroid orbital period (where secular terms are dominant) and is increasingly more accurate the further back from the impact event the manoeuvre is initiated. 

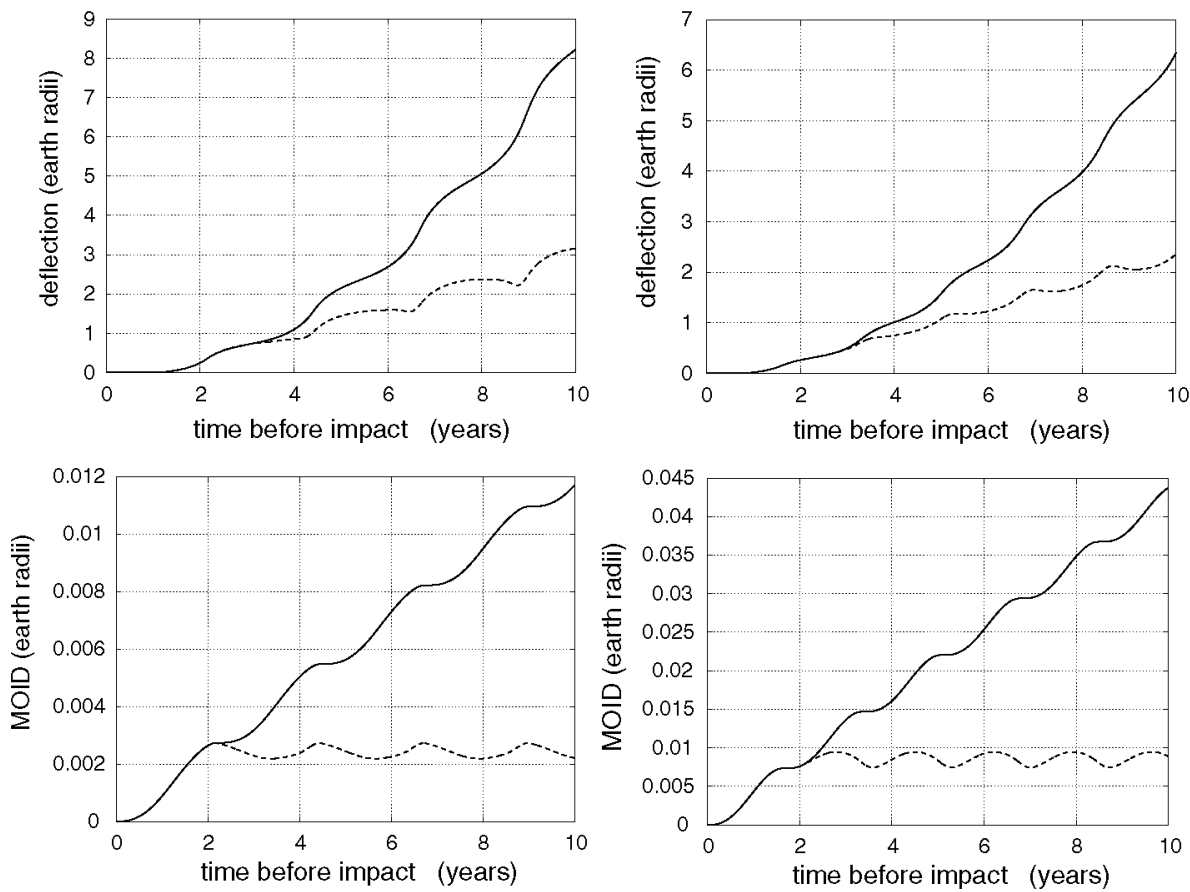

Fig. 2 B-plane deflection magnitude (upper row) and MOID (lower row) for asteroid $2007 \mathrm{VK}_{184}$ (left) and $2011 \mathrm{AG}_{5}$ (right) following a continuous tangential thrust of magnitude $1 \mathrm{~N}$. The abscissa represents the time before impact at which the manoeuvre starts. The thrust is applied continuously up to 10 years (solid line) or for up to 2 years and followed by a coast phase (dash line)

Fig. 3 Evolution of the asteroid position in the b-plane following a continuous 1-N thrust applied up to half an asteroid revolution prior to the scheduled impact

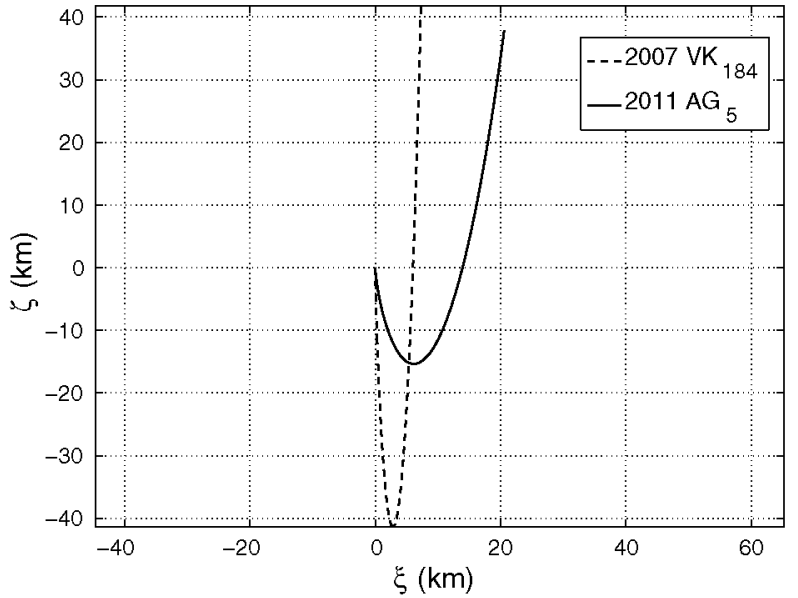



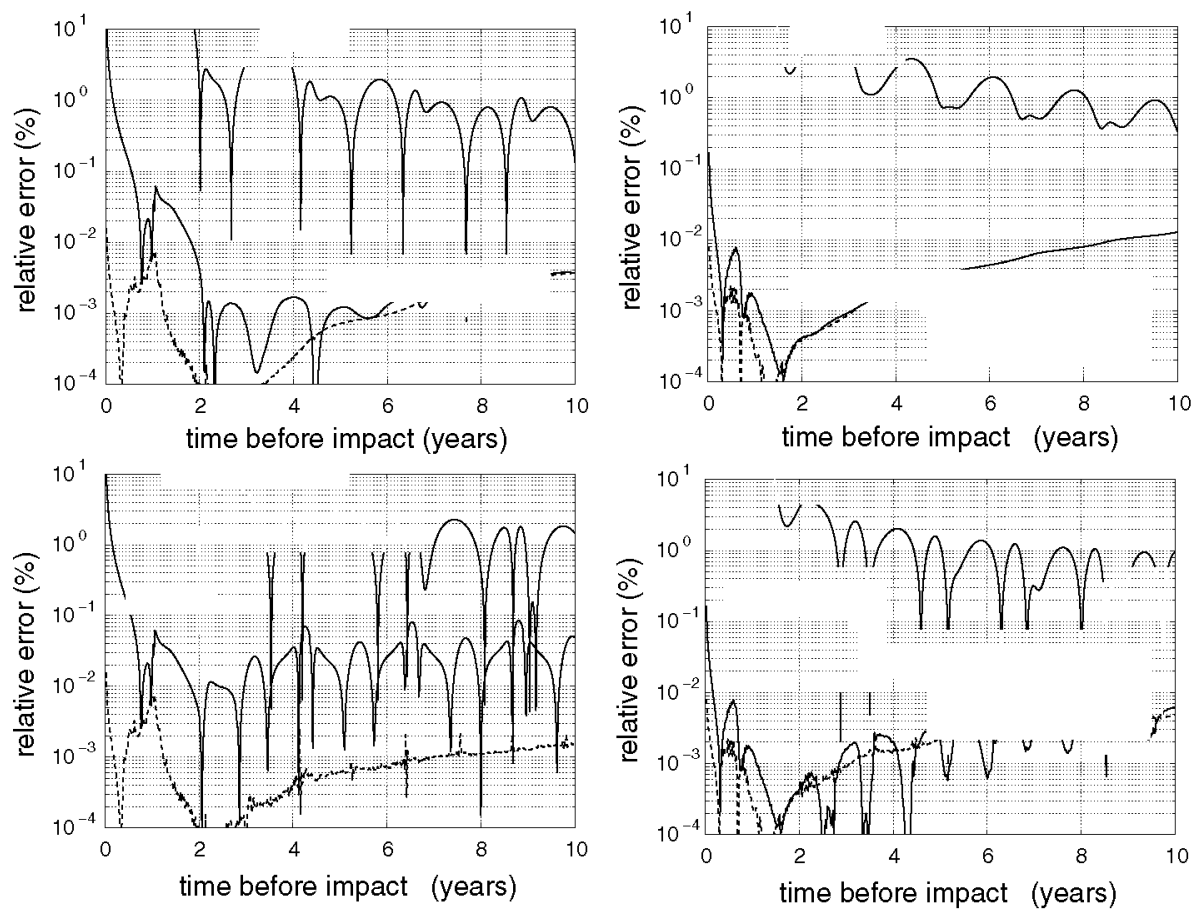

Fig. 4 Relative error on the b-plane deflection magnitude for asteroid $2007 \mathrm{VK}_{184}$ (left columm) and 2011 $\mathrm{AG}_{5}$ (right columm) employing the approximate analytical solution given by Eq. (55) (gray solid line) and the full analytical solution including oscillatory terms up to the 8th order (dark solid line) and 20th order (dash line) in the asteroid eccentricity. The same deflection strategy described in Fig. 2 is employed with the upper and lower column representing the full thrust and 2-years thrust case, respectively

\section{Conclusions}

The article provides accurate analytical expressions quantifying the impact b-plane position of an Earth-impacting asteroid after a deflection manoeuvre consisting of a constant tangential thrust phase followed by a coasting phase until the predicted impact event.

The deflection can be evaluated with very high accuracy (less than $0.02 \%$ even in the case of very short warning time) using a complete analytical expression including high-order oscillatory terms. A less accurate but compact analytical expression, accounting for secular terms only, allows estimating the total deflection with less than a few percent relative error if we exclude deflection manoeuvres starting less than one asteroid period before impact. This represents an improvement compared with previous work in which quadrature-based expressions were provided (Izzo 2007).

Deflection charts are computed for the case of asteroids $2007 \mathrm{VK}_{184}$ and $2011 \mathrm{AG}_{5}$, currently the only two NEOs with index 1 in the Torino scale, showing that a continuous $1-\mathrm{N}$ deflection thrust applied 10 years before impact and for a time span of 2 years is sufficient to deflect both asteroids by more than 2 Earth radii. An additional time span of typically 2-3 years accounting for the interplanetary transfer trajectory to rendezvous with the asteroid has also to be taken into account. 
Acknowledgments The work was supported by the DGI of the (former) Spanish Ministry Science and Innovation under the research grant "Dynamical Simulations of Complex Space Systems" contract number AYA2010-18796.

\section{Appendix I}

In the following we deal with the deflection computation for the particular case in which the asteroid and Earth heliocentric velocity vectors at the impact event are parallel. It can be easily verified that, under the hipothesis of circular orbit for the Earth, this is possible only when both the following conditions are verified:

1. the impact event occurs either at the apoapsis or periapsis of the asteroid orbit (i.e. $\alpha=0$ or $\alpha=\pi$ )

2. the asteroid orbit lies on the ecliptic plane $(i=0)$

From condition (1) and taking into account Eq. (3) we obtain the constraint $e=p-1$, which is substituted into Eqs. (17-19) to yield:

$$
\begin{aligned}
& \Delta x=-v_{\text {ast }} \Delta t, \\
& \Delta y=0, \\
& \Delta z=-\Delta r .
\end{aligned}
$$

From condition (1) and (2) Eq. (20) yields:

$$
\beta=0 \text {. }
$$

So the final deflection [Eqs. $(50,9)]$ results in:

$$
\delta=|\xi|=\Delta r,
$$

showing that in such circumstance the deflection magnitude coincides with the MOID and the asteroid is particularly difficult to deflect since the phasing component $v_{\text {ast }} \Delta t$ cannot be exploited. However, a quick inspection of Eq. (20) reveals that the angle $\beta$ is very sensitive to small variations of both $\alpha$ and $i$ leading to the conclusion that the case of $\beta \rightarrow 0$ is extremely unlikely and has little practical relevance.

\section{References}

Ahrens, T., Harris, A.: Deflection and fragmentation of near-earth asteroids. Nature 360(6403), 429-433 (1992) Battin, R.: An Introduction to the Mathematics and Methods of Astrodynamics. pp. 421 AIAA Educational Series, American Institute of Aeronautics and Astronautics, New York (NY) (1999)

Bombardelli, C., Peláez, J.: Ion beam shepherd for asteroid deflection. J. Guid. Control Dyn. 34(4), 12701272 (2011)

Bombardelli, C., Bau, G., Pelaez, J.: Asymptotic solution for the two-body problem with constant tangential thrust acceleration. Celest. Mech. Dyn. Astron. 110(3), 239-256 (2011)

Bonanno, C.: An analytical approximation for the moid and its consequences. Astron. Astrophys. 360, 411416 (2000)

Carusi, A.: Early neo deflections: a viable, lower-energy option. Earth Moon Planets 96(1), 81-94 (2005)

Carusi, A., Valsecchi, G., D'Abramo, G., Boattini, A.: Deflecting neos in route of collision with the earth. Icarus 159(2), 417-422 (2002)

Carusi, A., D'Abramo, G., Valsecchi, G.: Orbital and mission planning constraints for the deflection of neos impacting on earth. Icarus 194(2), 450-462 (2008)

Colombo, C., Vasile, M., Radice, G.: Semi-analytical solution for the optimal low-thrust deflection of nearearth objects. J. Guid. Control Dyn. 32(3), 796-809 (2009) 
Gong, S., Li, J., BaoYin, H.: Formation flying solar-sail gravity tractors in displaced orbit for towing near-earth asteroids. Celest. Mech. Dyn. Astron. 105(1), 159-177 (2009)

Izzo, D.: Optimization of interplanetary trajectories for impulsive and continuous asteroid deflection. J. Guid. Control Dyn. 30(2), 401-408 (2007)

Lu, E., Love, S.: Gravitational tractor for towing asteroids. Nature 438(7065), 177-178 (2005)

Melosh, H.: Solar asteroid diversion. Nature 366, 21-22 (1993)

Pelaez, J., Hedo, J., de Andres, P.: A special perturbation method in orbital dynamics. Celes. Mech. Dyn. Astron. 97(2), 131-150 (2007)

Scheeres, D., Schweickart, R.: The mechanics of moving asteroids. In: 2004 Planetary Defense Conference: Protecting Earth from Asteroids, pp. 23-26 (2004)

Song, Y., Park, S., Choi, K.: Optimal deflection of earth-crossing objects using a power limited spacecraft (aas 07-147). Adv. Astronaut. Sci. 127(1), 701 (2007)

Valsecchi, G., Milani, A., Gronchi, G., Chesley, S.: Resonant returns to close approaches: analytical theory. Astron. Astrophys. 408(3), 1179-1196 (2003)

Vasile, M., Maddock, C.: On the deflection of asteroids with mirrors. Celest. Mech. Dyn. Astron. 107(1), 265-284 (2010) 